\title{
Theory of local optical spectroscopy of quantum wires with interface fluctuations
}

\author{
Omar Di Stefano, ${ }^{\text {a) }}$ Salvatore Savasta, and Raffaello Girlanda \\ INFM and Dipartimento di Fisica della Materia e Tecnologie Fisiche Avanzate, Università di Messina, \\ Salita Sperone 31, I-98166 Messina, Italy
}

\begin{abstract}
We present a theory of local optical spectroscopy in quantum wires taking into account structural disorder. The calculated spatially resolved spectra show the individual spectral lines due to the exciton states localized by the disordered potential in agreement with experimental findings. We investigate systematically the influence of the potential profile and of the spatial resolution on the local optical spectra. Several line scans along the wire axis are obtained for different spatial correlations and strength of the disorder potential and for different spatial resolutions ranging from the subwavelength to the diffraction limit. Lowering the spatial resolution causes the disappearance of many spectral lines due to destructive spatial interference. However, our results show that information on the individual eigenstates of this quasi one-dimensional quantum system can be obtained at also resolutions significantly lower than the correlation length of interface fluctuations.
\end{abstract}

[DOI: $10.1063 / 1.1424049$ ]

\section{INTRODUCTION}

Over the past decade, considerable attention has been given to low-dimensional semiconductor structures, such as quantum wires (QWRs) and quantum dots, due to the effects of reduced dimensionality on the electronic density of states. Static structural disorder on the mesoscopic length scale is an unavoidable feature of such semiconductor nanostructures. In addition the typical sources of nonhomogeneity of bulk crystals, such as the impurities, lattice imperfections, etc., semiconductor quantum structures exhibit interface imperfections at different length scales. The effects of structural disorder on the optical and electronic properties of semiconductor nanostructures are very important as they substantially limit the performance of optoelectronic devices. ${ }^{1}$ In particular, disorder is detrimental in order to obtain sharp and controlled optical transitions in quantum structures and also high mobility modulation-doped heterostructures.

The optical spectra of semiconductor nanostructures, which in the absorption edges are dominated by excitons, yield valuable information on the quality of the interfaces and the growth process. ${ }^{2,3}$ The linear optical properties of excitons in such structures are very sensitive to structural disorder and thus provide a unique powerful tool both to characterize the quality of the quantum structures and to study many aspects of single-particle disordered quantum systems. Disorder in QWRs results in an effective onedimensional (1D) potential with spatial correlations which localize the center of mass of the exciton.

Photoluminescence (PL) and absorption spectroscopy appear as effective tools in order to get information on the quality of the nanostructures. The effective disorder potential gives rise to an asymmetric inhomogeneous broadening of

a) Author to whom correspondence should be addressed; electronic mail: distef@ortica.unime.it the exciton absorption and PL lines. However, the experimental values of the energy levels, transport length scales, and relaxation times obtained in this way are generally averaged over a larger distribution, reflecting intrinsic inhomogeneities between different emission sites as well as additional inhomogeneities arising from local environmental perturbations. The inability to resolve details within this distribution causes the lost of relevant information. In fact macroscopic optical probes perform a spatial averaging of spectral information providing insight into an inhomogeneous ensemble of quasi-0D localized exciton states. In order to investigate the optical properties and the effects of disorder on the electronic states of such heterostructures in detail, techniques of very high spatial resolution are needed.

Recently advanced techniques in optical spectroscopy allowed several groups to study individual exciton eigenstates which are laterally localized at the interface fluctuations. ${ }^{4-10}$ In particular, Hasen et al. ${ }^{6}$ reported spatially resolved PL images of excitons confined into an isolated gallium arsenide QWR. As the temperature of the structure is lowered, they observe a transition from a broad and continuous PL spectrum to a sharp set of emission peaks, which are spatially localized. More recently, the coexistence of localized and delocalized excitons in a quasi-1D GaAs QWR has been observed in luminescence experiments with high spectral and spatial resolution. ${ }^{11}$ These experimental findings show that when the spatial resolution approaches the scale either of quantum confinement or of the correlation lengths of interface fluctuations, scanning spectroscopy can be used to detect the spatial variations of the wave functions of the individual eigenstates. Stimulated by the relevance of the above experimental results, theoretical approaches modeling the interaction of quantum structures with highly inhomogeneous light fields have been recently presented. ${ }^{12-18}$

In this article, we present a systematic theory of the linear optical response of QWRs under the effect of highly 
inhomogeneous light fields and including structural disorder which is an unavoidable feature of real samples. We present a number of line scans along the free axis of different simulated wires. The theoretical calculations consist of two steps in close analogy with real experiments. The first step is the growth of the sample. Each sample corresponds to a single realization of disorder with given statistical properties. Once the samples have been obtained, we calculate the spectra by keeping the probe tip fixed at a point on the coordinate $Z$ along the wire. Each spectrum obtained with the probe tip at a fixed $Z$ is a single complete calculation.

\section{THEORY}

\section{A. The equation of motion}

According to the dynamics controlled truncation scheme, ${ }^{19}$ the relevant dynamical variable in the linear optical regime is the electron-hole $(e-h)$ pair amplitude $\hat{Y}_{\mathrm{eh}}$. Taking into account only a valence and a conduction subband, its real space expression is given by $\hat{Y}_{\text {eh }}$ $=\left\langle\hat{c}\left(z_{e}\right) \hat{h}\left(z_{h}\right)\right\rangle$, where $\hat{c}\left(z_{e}\right)$ and $\hat{h}\left(z_{h}\right)$ are the real space destruction operators, respectively, for the electron and the hole along the wire free axis. Starting from the Hamiltonian for the usual semiconductor model, including the spacedependent disorder potential and the dipole interaction with the light field, we obtain the following inhomogeneous timedependent Schrödinger equation for the $e-h$ amplitude:

$$
\left[i \hbar\left(\frac{\delta}{\delta t}\right)-\mathcal{H}_{\mathrm{eh}}\left(z_{e}, z_{h}\right)\right] Y_{\mathrm{eh}}\left(z_{e}, z_{h}\right)=-\mu \delta\left(z_{e}-z_{h}\right) E\left(z_{e}, t\right),
$$

where $E\left(z_{e}, t\right)$ is the input electric field at $z_{e}, \mu$ is the interband dipole moment, and

$$
\begin{aligned}
\mathcal{H}_{\mathrm{eh}}\left(z_{e}, z_{h}\right)= & -\frac{\hbar^{2}}{2 m_{e}} \nabla_{e}^{2}-\frac{\hbar^{2}}{2 m_{h}} \nabla_{h}^{2}+V_{d}^{e}\left(z_{e}\right)+V_{d}^{h}\left(z_{h}\right) \\
& +V_{\mathrm{eh}}\left(z_{e}, z_{h}\right)
\end{aligned}
$$

is the effective Hamiltonian operator, where $m_{e}$ and $m_{h}$ are the effective masses of the electron and the hole. Furthermore, $V_{\text {eh }}$ is the 1D Coulomb interaction

$$
V_{\mathrm{eh}}\left(z_{e}, z_{h}\right)=-\iint d \mathbf{x}_{e} d \mathbf{x}_{h} \frac{e^{2}\left|\chi_{e}\left(\mathbf{x}_{e}\right)\right|^{2}\left|\chi_{h}\left(\mathbf{x}_{h}\right)\right|^{2}}{\sqrt{\left|\mathbf{x}_{h}-\mathbf{x}_{e}\right|^{2}+\left(z_{e}-z_{h}\right)^{2}}},
$$

where $\mathbf{x} \equiv(x, y)$ and $\chi_{e(h)}(\mathbf{x})$ describes the two-dimensional confinement of the electron and the hole normal to the wire free axis. Finally, $V_{d}^{e}(z)$ is the $1 \mathrm{D}$ effective disordered potential for the electron

$$
V_{d}^{e}(z)=\int d \mathbf{x} V(\mathbf{r})\left|\chi_{e}(\mathbf{x})\right|^{2}
$$

where $V(\mathbf{r})$ is the $3 \mathrm{D}$ disordered potential arising from interface imperfections during the growth and/or patterning processes.

\section{B. The center of mass (COM) motion}

In reasonable good quality quantum structures the amplitude of the confinement energy fluctuations is typically 1 order of magnitude smaller than the exciton binding energy. ${ }^{20}$ In this limit, the relative exciton motion described by the effective wave function $\phi\left(z_{e}-z_{h}\right)$ may be assumed to be undistorted by disorder. Disorder significantly affects only the COM motion through an effective potential $V(z)$, where $z$ now indicates the COM vector lying along the wire free axis. The COM coordinate is given by

$$
z=\frac{m_{h}^{*} z_{h}+m_{e}^{*} z_{e}}{M}
$$

where $M=m_{e}^{*}+m_{h}^{*}$ is the exciton kinetic mass, and

$$
\rho=z_{h}-z_{e}
$$

is the relative coordinate. Introducing the new variables, the Hamiltonian in Eq. (2) becomes

$$
\mathcal{H}_{\mathrm{eh}}(\rho, z)=-\frac{\hbar^{2}}{2 M} \nabla_{z}^{2}-\frac{\hbar^{2}}{2 \mathcal{M}} \nabla_{\rho}^{2}+V_{C}(\rho)+V_{d}(\rho, z),
$$

where $\mathcal{M}=m_{e}^{*} m_{h}^{*} /\left(m_{e}^{*}+m_{h}^{*}\right)$ is the reduced electron-hole mass. We observe that the wave functions describing the relative motion $\phi_{n}(\rho)$ are the solution of the Schrödinger equation

$$
\left[-\frac{\hbar^{2}}{2 \mathcal{M}} \nabla_{\rho}^{2}+V_{C}(\rho)\right] \phi_{n}(\rho)=\hbar \omega_{n} \phi_{n}(\rho) .
$$

Thus we can expand the $e-h$ amplitude as

$$
Y_{\mathrm{eh}}(\rho, z)=\sum_{n} \phi_{n}(\rho) \mathcal{Y}_{n}(z)
$$

or, in Dirac notation,

$$
\left\langle\rho, z \mid Y_{\mathrm{eh}}\right\rangle=\sum_{n}\left\langle\rho \mid \phi_{n}\right\rangle\left\langle z \mid \mathcal{Y}_{n}\right\rangle
$$

Taking the time Fourier transform of Eq. (1), multiplying by $\phi_{n}^{*}(\rho)$, integrating in the relative space [in other words, projecting Eq. (7) in the relative exciton $n$-subspace], and using Eq. (8), straightforward calculations allow us to write

$$
\begin{aligned}
\sum_{n^{\prime}} & {\left[\left(\omega+i \gamma-\hbar \omega_{n}\right) \delta_{n, n^{\prime}}-\mathcal{H}_{n, n^{\prime}}(z)\right] \mathcal{Y}_{n^{\prime}}(z) } \\
= & -\mu \phi_{n}^{*}(0) E(z)
\end{aligned}
$$

where

$$
\mathcal{H}_{n, n^{\prime}}(z)=-\frac{\hbar^{2}}{2 M} \nabla_{z}^{2} \delta_{n, n^{\prime}}+V_{n, n^{\prime}}^{d}(z)
$$

with

$$
V_{n, n^{\prime}}^{d}(z)=\int d \rho \phi_{n}^{*}(\rho) V_{d}(\rho, z) \phi_{n^{\prime}}(\rho) .
$$

In Eq. (11), we added $\gamma$ to describe the homogeneous broadening. As shown by Eq. (12), disorder causes mixing between the levels of the relative exciton space. We consider the usual case with the $1 s$ exciton binding energy larger than the disordered potential, so that we can neglect the mixing, 
focusing our attention only on the lowest bound state $1 s$. Equation (11) for the lowest bound state (which dominates the band gap optical response) can thus be written as

$$
[\omega+i \gamma-\mathcal{H}(z)] \mathcal{Y}(z)=-\mu \phi_{n}^{*}(0) E(z, \omega),
$$

where

$$
\mathcal{H}(z)=-\frac{\hbar^{2}}{2 M} \nabla_{z}^{2}+\hbar \omega_{1 s}+V_{d}^{\mathrm{eff}}(z),
$$

with

$$
V_{d}^{\mathrm{eff}}(z)=\int d \rho\left|\phi_{1 s}(\rho)\right|^{2} V_{d}(\rho, z) .
$$

Equations (14) and ( 15) show that the wire excitons generated by a low optical excitation behave as an effective quantum particle of mass $M$ which moves on a line under the effect of an effective disorder potential. Equation (14) can be written formally as

$$
(\omega+i \gamma-\hat{\mathcal{H}})|\mathcal{Y}\rangle=-\mu \phi_{n}^{*}(0)|E(\omega)\rangle,
$$

where, of course, the operators and the vectors operate in the COM space.

\section{Local optical spectroscopy}

The wire interband polarization density can be expressed as

$$
P^{-}(z) \equiv\left\langle\hat{P}^{-}(z)\right\rangle=\left\langle\left[\hat{P}^{+}(z)\right]^{\dagger}\right\rangle=\mu\left\langle\hat{h}^{\dagger}(z) \hat{c}^{\dagger}(z)\right\rangle .
$$

Taking into account only the contribution from the $1 s$ exciton, we obtain in the COM approximation

$$
P^{+}(z)=\mu Y(\rho=0, z)=\mu \phi_{1 s}(0) \mathcal{Y}(z) .
$$

From Eq. (14), we obtain the following equation for the interband polarization:

$$
[\omega+i \gamma-\mathcal{H}(z)] P^{+}(z)=-\mu^{2}\left|\phi_{1 s}(0)\right|^{2} E(z) .
$$

The polarization can thus be obtained inverting Eq. (20),

$$
P^{+}(z)=\int d z^{\prime} \chi\left(z, z^{\prime}\right) E\left(z^{\prime}\right)
$$

with the nonlocal susceptibility given by

$$
\chi\left(z, z^{\prime}\right)=-\mu^{2}\left|\phi_{1 s}(0)\right|^{2}\left\langle z\left|(\omega+i \gamma-\hat{\mathcal{H}})^{-1}\right| z^{\prime}\right\rangle .
$$

Therefore, the excitonic polarization $P(z, \omega)$ is thus driven by the electric field through the nonlocal susceptibility tensor, $\chi\left(z, z^{\prime}\right)^{21,22}$ according to the general linear response theory. ${ }^{23}$

Equation (21) gives the wire polarization under the effect of a possibly nonuniform illumination. In the following, we consider a highly inhomogeneous input light field with a given profile $g$ centered around the beam position $Z$

$$
E_{Z}(z, \omega)=E(\omega) g(z-Z) .
$$

From classical electromagnetism, the total absorption under local illumination is proportional to ${ }^{15}$

$$
S^{I}(Z)=\operatorname{Im} \int d z E_{Z}^{*}(z) P(z) \equiv \operatorname{Im}\langle\hat{E}(\omega) \mid \hat{P}(\omega)\rangle .
$$

Accordingly, we can also define a local absorption coefficient which, of course, is a function of the beam position and shape

$$
\alpha_{g}(Z, \omega)=\frac{\operatorname{Im}\langle\hat{E}(\omega) \mid \hat{P}(\omega)\rangle}{\langle\hat{E}(\omega) \mid \hat{E}(\omega)\rangle} .
$$

We obtain

$$
\alpha_{g}(Z, \omega)=\left\langle g_{Z}\left|\frac{1}{\omega+i \gamma-\hat{\mathcal{H}}}\right| g_{Z}\right\rangle,
$$

where $\left\langle z \mid g_{Z}\right\rangle=g(z-Z)$. Significant, although not direct, information on absorption under local illumination can also be obtained by subwavelength excitation spectroscopy. ${ }^{8,24}$ In real space Eq. (26) can be written as

$$
\alpha_{g}(Z, \omega)=\iint d Z d Z^{\prime} g(Z) \chi\left(Z, Z^{\prime}, \omega\right) g\left(Z^{\prime}\right) \text {. }
$$

\section{Realization of the disorder potential}

The effective disorder potential felt by excitons is a result of a 2D integration of the actual 3D disorder potential of the nanostructure weighted by the $2 \mathrm{D}$ electron and hole confinement wave functions [see Eq. (4)] followed by a convolution with the $1 s$ exciton wave function according to Eq. (16). We model the $1 \mathrm{D}$ random potential $V(z)$ felt by the wire excitons as a zero mean, Gauss distributed and spatially correlated process defined by the property

$$
\left\langle V(z) V\left(z^{\prime}\right)\right\rangle=v_{0}^{2} e^{-\left|z-z^{\prime}\right|^{2} / 2 \xi^{2}},
$$

where $\langle\ldots\rangle$ denotes the ensemble average over random configurations, $v_{0}$ is the width of the energy distribution, and $\xi$ is the correlation length characterizing the potential fluctuations. If, as a consequence of the convolution, the correlation length of the potential fluctuations is smaller than the exciton Bohr radius, $\xi$ can be assumed to be on the order of the Bohr radius. Realistic random potentials can be rather different from the model adopted here. However this widely adopted model ${ }^{25}$ retains the main physical aspects in a very simple way.

\section{NUMERICAL RESULTS}

In this section, we present the results of numerical calculations of the local absorption spectra $\alpha_{g}(Z, \omega)$. In particular, we investigate systematically the influence of both the potential profile and spatial resolution on the local optical spectra reporting several line scans along the wire axis obtained for different spatial correlations and strength of the disorder potential and for different spatial resolutions ranging from subwavelength to diffraction limit.

Simulations are carried out in real space, mapping the Hamiltonian on a fine mesh of points. The tridiagonalization of the Hamiltonian has been performed using the Lanczos algorithm, starting from the normalized initial state $\left|g_{z}\right\rangle$. The relevant spectrum is obtained by the inversion of the resolvent matrix $^{17}(\omega+i \gamma-H)^{-1}$, which is a very fast procedure using the tridiagonal form of the Hamiltonian $H$. We adopt an exciton kinetic mass of $m=0.25 m_{0}$ typical for AlAs/ 


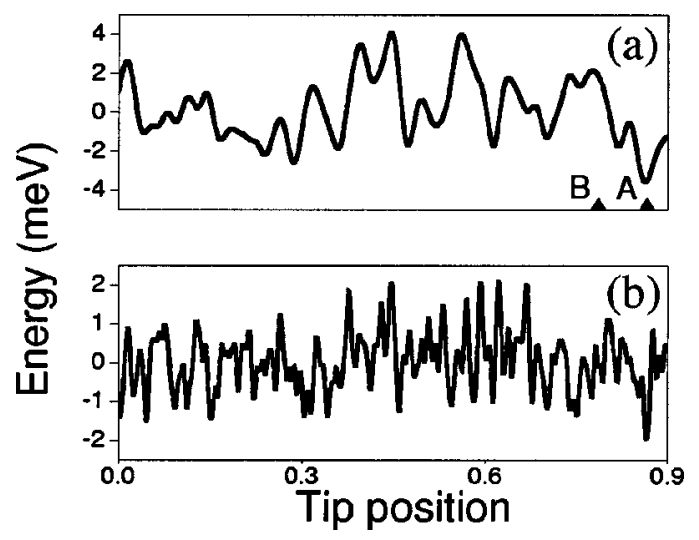

FIG. 1. Two different realization of disorder potential with different correlation length $\xi$ : (a) sample with $\xi=30 \mathrm{~nm}$ and (b) sample with $\xi=8 \mathrm{~nm}$.

GaAs wires. We have considered a system size of $L=6 \mu \mathrm{m}$ which has been divided into 3000 steps. We have adopted periodic boundary conditions in order to avoid spurious reflection at the boundaries. These boundary conditions make the Hamiltonian matrix not perfectly tridiagonal. The eigenstates of this quantum system are characterized by an individual broadening $\gamma$, which is due mainly to the acoustic phonons scattering and radiative decay. They both depend on the specific eigenfunction of the state. ${ }^{20}$ Typical values for AlAs/GaAs systems are below $80 \mu \mathrm{eV}{ }^{4,24}$ The calculation of these broadenings is not the aim of this work. All calculations presented here have been performed by using a fixed value of $\gamma=80 \mu \mathrm{eV}$ which could be interpreted as the resolution limit of the spectrometer.

Figure 1 shows two realizations of disordered potential obtained by using two different correlation lengths. In Fig. 1(a) we used $\xi=30 \mathrm{~nm}$ and $v_{0}=1.73 \mathrm{meV}$; in Fig. 1(b) $\xi$ $=8 \mathrm{~nm}$ and $v_{0}=0.67 \mathrm{meV}$. These two potential realizations represent the starting point of subsequent calculations and have been displayed in the region that will be probed in the following by calculations of local optical spectra. Figure 2 shows spatially resolved spectra $\alpha_{g}(Z, \omega)$ calculated with the beam position centered at two different locations and by using beams of different spatial extension, as indicated in the

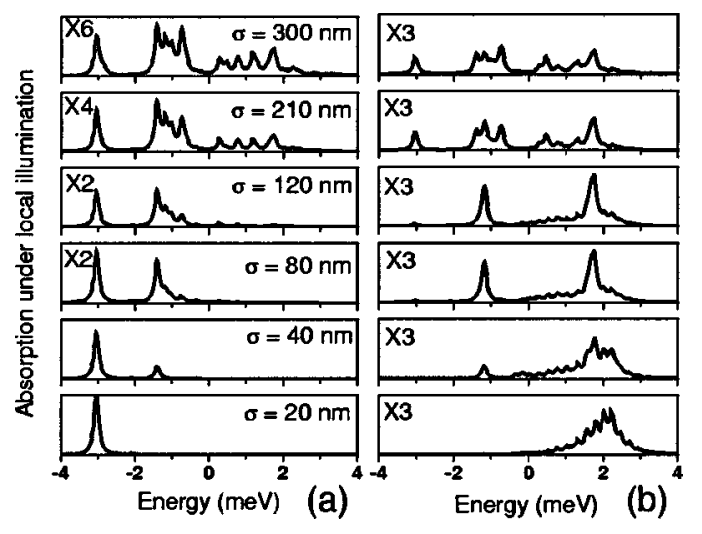

FIG. 2. Spatially resolved spectra for the first sample [Fig. 1(a)] calculated on the potential well $A$ and on the barrier $B$ [see labels in Fig. 1(a)]. The beams used are of different spatial extension $\sigma$ as indicated in the insets.

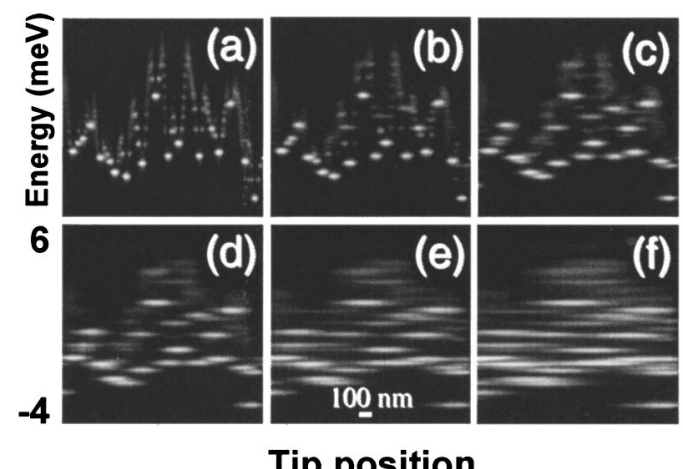

FIG. 3. Local absorption $\alpha_{g}(Z, \omega)$ as a function of beam position $Z$ and photon energy along the free $z$ axis of the wire, obtained for the first sample [Fig. 1(a)] using different spatial resolutions: (a) $\sigma=20 \mathrm{~nm}$, (b) $\sigma=40 \mathrm{~nm}$, (c) $\sigma=80 \mathrm{~nm}$, (d) $\sigma=120 \mathrm{~nm}$, (e) $\sigma=210 \mathrm{~nm}$, and (f) $\sigma=300 \mathrm{~nm}$. The energy range on the vertical axis is between -4 and $6 \mathrm{meV}$ for all the panels.

figure. In particular, Fig. 2(a) has been obtained centering the beam at the potential local minimum $A$ indicated in Fig. 1(a). At very high spatial resolution $(\sigma=20 \mathrm{~nm})$ the spectrum displays an isolated sharp peak originating from the lowest exciton state localized at the local minimum $A$. At $\sigma=40 \mathrm{~nm}$ a second peak from a higher energy state appears. As the resolution is lowered, the contribution from the lowest exciton state remains clearly visible and a quasicontinuum absorption band appears. Figure 2(b) has been obtained centering the beam at the potential barrier $B$ indicated in Fig. 1(a). In contrast to Fig. 1(a), at very high spatial resolution ( $\sigma$ $=20 \mathrm{~nm}$ ) the spectrum displays a quasicontinuous band originating from higher energy states. Lowering the spatial resolution, the spectrum evolves assuming features that are analogous to those of the corresponding spectra in Fig. 1(a). We also notice that the contribution from the lowest exciton state appears. Figure 2 gives a first indication of the information that can be gained by local optical spectra and of the impact that spatial resolution has on these spectra. In particular, Fig. 2 shows how, at subwavelength spatial resolution, local optical spectra taken at different points can be completely different.

The results shown in Fig. 2 do not provide any information on the spatial extent of exciton states. A more detailed analysis can be achieved by scanning spectroscopy. Figure 3 displays spatially resolved spectra $\alpha_{g}(Z, \omega)$ as a function of photon energy $\omega$ and beam position $Z$ along the free $z$ axis of the wire. These line scans, obtained by using beams with different spatial extension, have been calculated for the sample of Fig. 1(a). At high spatial resolution (a), (b) it is possible to observe sharp and spatially narrow structures from the individual eigenstates of the quantum system, where these structures provide detailed information on the spatial extension of the optically active quantum states. In particular, these plots clearly show the sharp peaks coming from the lowest states of COM motion, localized close to the local minima of the potential, as well as the excited states. At low energy, as expected, the structures at a given energy are localized inside only one potential well. By increasing the 


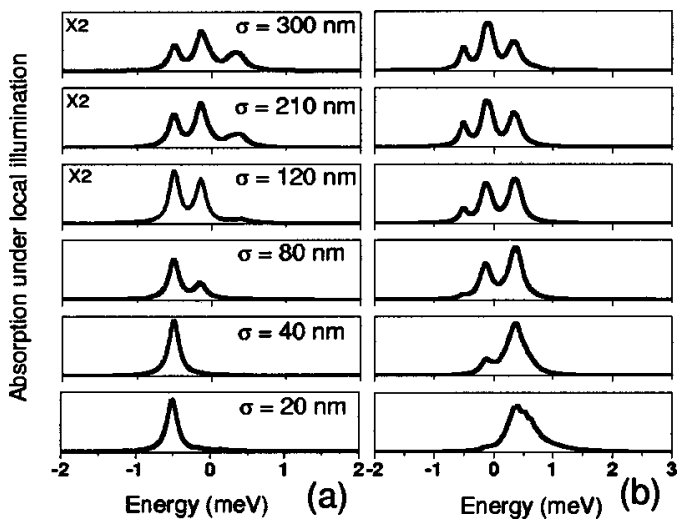

FIG. 4. Spatially resolved spectra as in Fig. 2 for a sample obtained reducing by a factor 5 the potential of Fig. 1(a).

energy, it is possible to observe structures which extend over different wells, arising from tunneling states of coupled potential wells, in agreement with spatially resolved measurements in QWRs. ${ }^{11}$ It is quite interesting to observe that even the states on the top of the barriers are spatially localized, although they give rise to a quasicontinuous absorption band [see Fig. 2(b)]. Comparison with the corresponding disorder potential [Fig. 1(a)] shows that these high-resolution line scans mirror the potential profile, as it would be in the classical limit. This classical limit holds when the local energy variation $v_{0}$ largely exceeds the energy $E_{c}=\hbar^{2} / 2 M \xi^{2}$ needed to localize the exciton within $\xi$. The potential in Fig. 1 (a) is characterized by a ratio $v_{0} / E_{c} \approx 10$. Lowering the spatial resolution, the structures broaden and information on the potential profile is partially lost. However, we notice that even when using a beam extension of $300 \mathrm{~nm}$, well beyond the spatial correlation of the disorder potential, a number of individual structures remain visible, in agreement with experimental findings. ${ }^{6}$ The relative intensity of the sharp lines is extremely sensitive to spatial resolution. Increasing the spatial extension of the probe, some structures tend to disappear, while other structures increase their intensity. Cancellation effects are due to destructive spatial interference of the corresponding quantum state and illustrate both the nonlocal character of light-matter interaction in semiconductors [see Eqs. (21) and (27)] and the influence that the phase of wave functions may have on the local optical properties. ${ }^{15,18}$

In Fig. 4, we show spatially resolved spectra calculated for a sample obtained simply by reducing the potential in Fig. 1(a) by a factor $5\left(v_{0}=0.35\right)$. By lowering the height of the potential wells, we observe a reduction of the spectral region, in agreement with what is generally observed in global spectra. We also observe a reduction of the excited states and an overall broadening of the structures. By lowering the spatial resolution, the number of individual structures that remain distinct is largely reduced, as can be seen in comparison with Fig. 3. Figure 5 shows spatially resolved spectra calculated with the beam position centered at the two different locations already chosen for Fig. 2.

The results shown until now were obtained using a disordered potential with a quite large correlation length $(\xi$ $=30 \mathrm{~nm}$ ). In this case, the structures in the spatially resolved

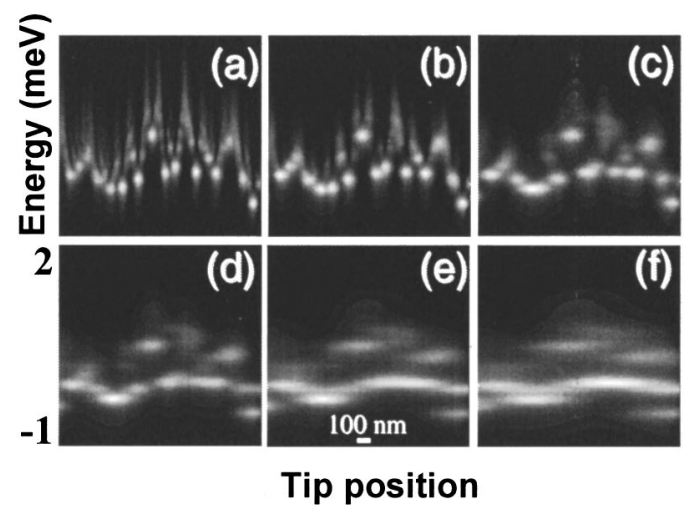

FIG. 5. Local absorption $\alpha_{g}(Z, \omega)$ as a function of beam position $Z$ and photon energy along the free $z$ axis of the wire, obtained for the same potential of Fig. 1(a) reduced by a factor 5 and using different spatial resolutions: (a) $\sigma=20 \mathrm{~nm}$, (b) $\sigma=40 \mathrm{~nm}$, (c) $\sigma=80 \mathrm{~nm}$, (d) $\sigma=120 \mathrm{~nm}$, (e) $\sigma=210 \mathrm{~nm}$, and (f) $\sigma=300 \mathrm{~nm}$. The energy range on the vertical axis is between -1 and $2 \mathrm{meV}$ for all the panels.

spectra follow quite closely, almost classically, the disordered potential giving precise information on the potential fluctuations of the sample [see Figs. 1(a) and 3(a)]. In the next step, we approach the quantum-mechanical limit by performing calculations on a sample with the potential shown in Fig. 1(b), characterized by a ratio $v_{0} / E_{c} \approx 0.3$. In this case (see Fig. 6), even the lowest energy states of the quantum system appear to be more extended than the potential correlation length. While information on the potential profile is lost, Figs. 6(a) and 6(b) show that even in this case, toward the quantum mechanical limit, subwavelength spectroscopy is able to provide detailed and complete information on the individual eigenstates of the quantum system. Figure 6(a) shows a quite different space-energy distribution of structures from that in Fig. 3(a). Looking carefully at Fig. 6(a), direct evidence of quantum mechanical level repulsion [not present in Fig. 3(a)] can be inferred. In random quantum mechanical systems the eigenvalues are not simply random numbers, but are correlated via the typical quantum mechani-

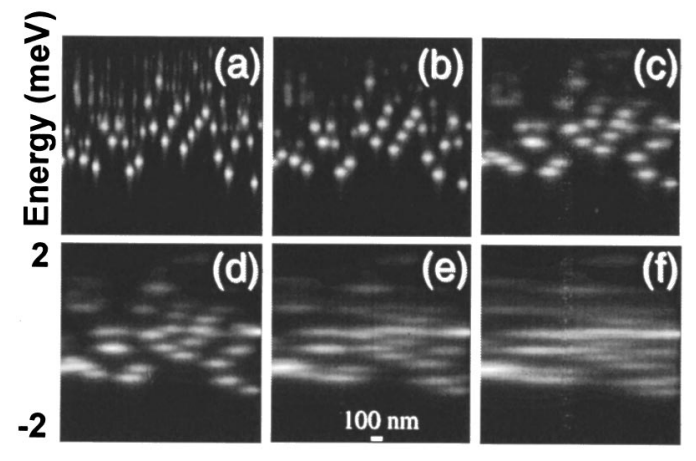

Tip position

FIG. 6. Local absorption $\alpha_{g}(Z, \omega)$ as a function of beam position $Z$ and photon energy along the free $z$ axis of the wire, obtained for the third sample of Fig. 2(a) using different spatial resolutions: (a) $\sigma=20 \mathrm{~nm}$, (b) $\sigma=40 \mathrm{~nm}$, (c) $\sigma=80 \mathrm{~nm},($ d) $\sigma=120 \mathrm{~nm}$, (e) $\sigma=210 \mathrm{~nm}$, and (f) $\sigma=300 \mathrm{~nm}$. The energy range on the vertical axis is between -2 and $2 \mathrm{meV}$ for all the panels. 
cal level repulsion. Quantum mechanical levels repel each other only if they interact, i.e., in this case, if their wave function spatially overlap.

\section{CONCLUSION}

In summary, we presented a theoretical formulation of scanning local optical spectroscopy in disordered quantum structures. We performed calculations for three different samples. The simulations, displayed as a function of position and photon energy, are shown for different spatial resolutions ranging from subwavelength to diffraction limit. These simulations show that, at realistic spatial resolution and approaching the scale of the correlation lengths of interface fluctuations, scanning near-field spectroscopy can look at the spatial variations of the wave functions of the individual eigenstates of the quantum system. When the spatial resolution is lowered many lines disappear because of the destructive spatial interference of quantum states. The calculated scans show that information can be obtained about the quasionedimensional quantum states determined by the disordered potential, even at resolutions well beyond the correlation length of interface fluctuations. Our approach can be easily extended to more realistic disordered potentials, describing the actual shape of monolayer-high islands.

The results presented in this work have been obtained assuming that disorder does not perturb the $e-h$ relative motion. Work is in progress in order to relax this approximation. We observe that in narrow quantum structures the inhomogeneous linewidth can be comparable to the exciton binding energy. In this case this approximation becomes questionable. Going beyond the approximation implies the study of two opposite charged interacting particles in a disordered landscape and under local illumination. This study also would allow analysis of the behavior of the exciton continuum in the presence of both disorder and local illumination. A further important extension of this theory is the inclusion of the interaction with the phonon bath. This would allow the microscopic calculation of emission spatially resolved spectra that are more easily accessible to experiments.

\section{ACKNOWLEDGMENT}

The authors acknowledge the support of INFM through the project NanoSNOM.

${ }^{1}$ J. Christen, in Festkörperprobleme, Advances in Solid State Physics, edited by P. Große (Pergamon, Braunschweig, 1990), Vol. 30, p. 239.

${ }^{2}$ C. Weisbuch, R. Dingle, A. C. Grossard, and W. Wiegmann, Solid State Commun. 37, 219 (1981).

${ }^{3}$ M. A. Herman, D. Bimberg, and J. Christen, J. Appl. Phys. 70, R1 (1991).

${ }^{4}$ H. F. Hess, E. Betzig, T. D. Harris, L. N. Pfeiffer, and K. W. West, Science 264, 1740 (1994).

${ }^{5}$ A. Zrenner, L. V. Butov, M. Hagn, G. Abstreiter, G. Böhm, and G. Weinmann, Phys. Rev. Lett. 72, 3382 (1994).

${ }^{6}$ J. Hasen, L. N. Pfeiffer, A. Pinczuk, S. He, K. W. West, and B. S. Dennis, Nature (London) 390, 54 (1997).

${ }^{7}$ D. Gammon, E. S. Snow, B. V. Shanabrook, D. S. Katzer, and D. Park, Science 273, 87 (1996).

${ }^{8}$ D. Gammon, E. S. Snow, B. V. Shanabrook, D. S. Katzer, and D. Park, Phys. Rev. Lett. 76, 3005 (1996).

${ }^{9}$ A. Gustafsson, M. E. Pistol, L. Montelius, and L. Samuelson, J. Appl. Phys. 84, 1715 (1998).

${ }^{10}$ V. Emiliani, Ch. Lienau, M. Hauert, G. Col, M. De Giorgi, R. Rinaldi, A. Passaseo, and R. Cingolani, Phys. Rev. B 60, 13335 (1999).

${ }^{11}$ F. Intonti, V. Emiliani, C. Lienau, T. Elsäesser, R. Näotzel, and K. H. Ploog, Phys. Rev. B 63, 075313 (2001).

${ }^{12}$ E. Runge and R. Zimmermann, Phys. Status Solidi B 206, 167 (1998).

${ }^{13}$ B. Hanewinkel, A. Knorr, P. Thomas, and S. W. Koch, Phys. Rev. B 55, 13715 (1997).

${ }^{14}$ G. W. Bryant, Appl. Phys. Lett. 72, 768 (1998).

${ }^{15}$ O. Mauritz, G. Goldoni, F. Rossi, and E. Molinari, Phys. Rev. Lett. 82, 847 (1999).

${ }^{16}$ O. Mauritz, G. Goldoni, E. Molinari, and F. Rossi, Phys. Rev. B 62, 8204 (2000).

${ }^{17}$ S. Savasta, G. Martino, and R. Girlanda, Phys. Rev. B 61, 13852 (2000).

${ }^{18}$ O. Di Stefano, S. Savasta, G. Martino, and R. Girlanda, Phys. Rev. B 62, 11071 (2000), Appl. Phys. Lett. 77, 2804 (2000).

${ }^{19}$ K. Victor, W. M. Axt, and A. Stahl, Phys. Rev. B 51, 14164 (1995).

${ }^{20}$ R. F. Schnabel, R. Zimmermann, D. Bimberg, H. Nickel, R. Lösch, and W. Schlapp, Phys. Rev. B 46, 9873 (1992).

${ }^{21}$ A. D'Andrea and R. Del Sole, Phys. Rev. B 25, 3714 (1982).

${ }^{22}$ K. Cho, J. Phys. Soc. Jpn. 55, 4113 (1986).

${ }^{23}$ R. Kubo, J. Phys. Soc. Jpn. 12, 570 (1957).

${ }^{24}$ Q. Wu, R. D. Grober, D. Gammon, and D. S. Katzer, Phys. Rev. Lett. 83, 2652 (1999).

${ }^{25}$ S. Glutsch and F. Bechstedt, Phys. Rev. B 50, 7733 (1994). 